\title{
Synthesis and in vitro biological effect of GnRH-protoporplyrin IX conjugates
}

\author{
Lilla Pethó1 ${ }^{1}$,ózsef Murányi², Nassima Kram¹, Gyöngyi Bökönyi², Gabriella Csík ${ }^{3}$, Gábor Mezó ${ }^{1}$ \\ ${ }^{1}$ MTA-ELTE Research Group of Peptide Chemistry, Eötvös Loránd University, Faculty of Science, Institute of Chemistry, Hungary \\ ${ }^{2}$ MTA-SE Pathobiochemistry Research Group, Hungary \\ ${ }^{3}$ SE Department of Biophysics and Radiation Biology, Hungary
}

https://doi.org/10.17952/35EPS.2018.317

Photodynamic therapy (PDT) combines non-toxic components, a photosensitizer (PS), light and oxygen. If the photosensitizer is activated to its excited states by irradiation with visible light in the presence of molecular oxygen, electron and energy transfers can produce reactive oxygen species (ROS) in the tissue. These ROS react rapidly with the biomolecules leading to cell death via apoptosis or necrosis. In most cases a singlet oxygen $\left({ }^{1} \mathrm{O}\right)$ is generated that has a short intracellular life time $(3 \mu \mathrm{s})$ and a very small intracellular diffusion distance that makes PDT highly selective. Unfortunately, photosensitizers accumulate in healthy tissues too, causing severe side effects like prolonged skin and eye photosensitivity. Therefore, the conjugation of the PS to a carrier peptide that itself has anti-tumor effect can increase the efficacy and selectivity of the treatment.

Protoporphyrin IX (PPIX) is a second-generation PS, it is non-toxic without irradiation, but can efficiently absorb light in the visible area. $635 \mathrm{~nm}$ irradiation is usually used to reach deeper $(3 \mathrm{~mm})$ penetration. PpIX has two carboxyl groups that are suitable for conjugation to targeting moieties.[1]

Gonadotropin-releasing hormone $(\mathrm{GnRH})$ receptors are overexpressed on several tumor cells, e.g. on tumors of the reproductive organs or on oral and laryngeal cancer cells, which makes this receptor a proper

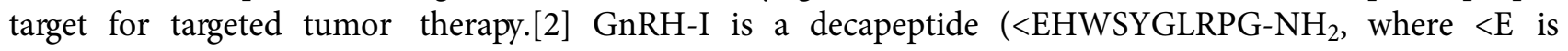
pyroglutamic acid) synthesized and released in the hypothalamus that plays a central role in the vertebrate reproduction by regulating gonadal activity.[3] Several different isoforms were isolated from different

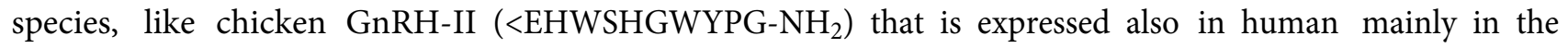
kidneys, bone marrow and prostate, and is found to be a neuromodulator that stimulates sexual behavior[4] or GnRH-III ( $<$ EHWSHDWKPG- $\left.\mathrm{NH}_{2}\right)$ that was originally isolated from sea lamprey.

GnRH-III binds to both type I and type II GnRH receptors and inhibits proliferation of different cancer cells while having insignificant hormonal activity.[5]

The sequences show that the $\mathrm{N}$ - and C- terminal parts are conserved, but the amino acids 5-8 can be changed without significant loss of efficacy.[4] In the sequence of GnRH-I and GnRH-II, the glycine in position 6 can be replaced by D-lysine, which serves as conjugation site, increases enzymatic stability and enhances the agonistic effect too.[6] Rahimipour et al. have already conjugated PpIX to GnRH-I[6DLys], and the selective receptor mediated phototoxicity could be demonstrated on T3-1 pituitary gonadotrope cell line.[7]

In most isoform the serine in position 4 can be replaced by a butyric acid modified lysine (Lys $(\mathrm{Bu}))$ since this change increases the receptor binding affinity and the stability of the molecule against enzymes. [8]

In this work GnRH-protoporphyrin IX conjugates were synthesized using various $\mathrm{GnRH}$ analogues. All peptides were synthesized manually using solid phase peptide synthesis according to standard Fmoc/tBu strategy. GnRH-I and GnRH-II analogues modified with butyrylated lysin (Lys(Bu)) in position 4 were synthesized with using Fmoc-Lys(Dde)-OH. After finishing the protected decapeptides, Dde group of ${ }^{4}$ Lys was removed on the resin and the acylation was performed by using butyric anhydride and DIPEA. The GnRH-III analogue was synthesized with aspartic acid methyl ester $(\mathrm{Asp}(\mathrm{OMe}))$ in position 6 since the free carboxylic acid could have interfered with the PpIX conjugation. The methyl ester group is labile under basic conditions, so in this case Fmoc-Lys(Mtt)-OH was incorporated into the peptide sequence instead of the Dde protected lysine and the butyrylation was performed after the Mtt removal. 

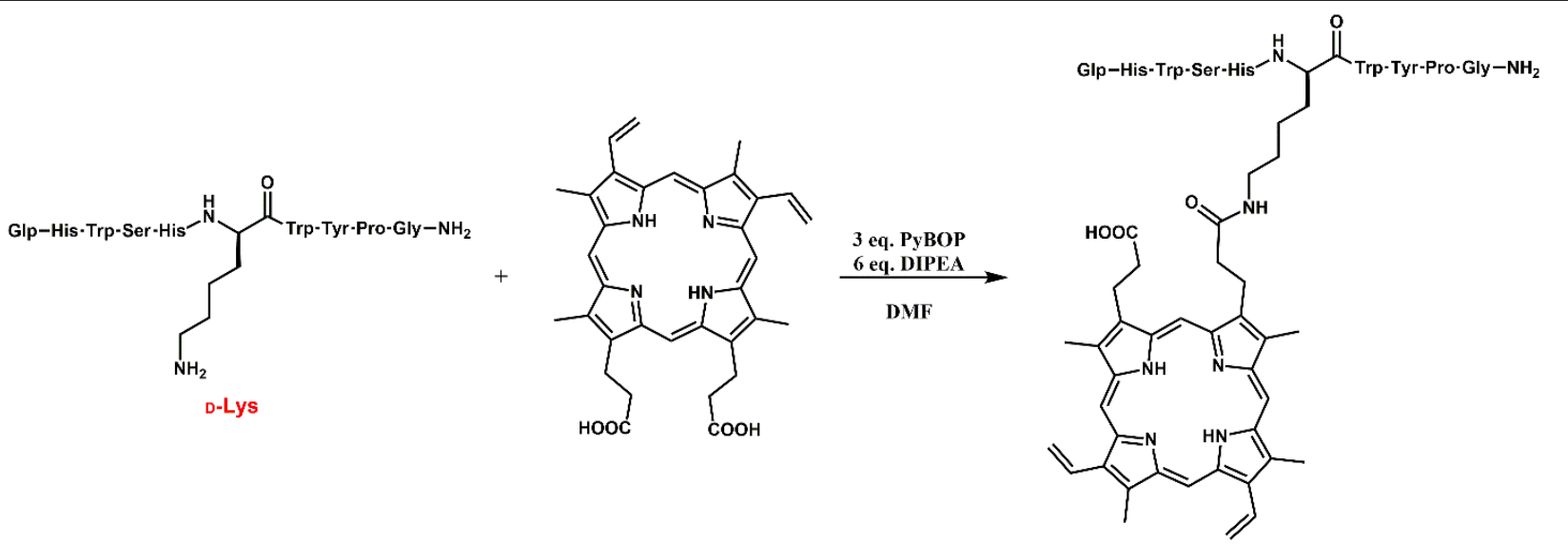

Figure 1: Synthesis of the GnRH-I - PpIX conjugate via amide bond formation

The purified peptides were conjugated to PpIX via an amide bond in solution phase using PyBOP (Figure 1).

The in vitro biological assays were performed on Detroit-562 human pharynx carcinoma cells that highly express GnRH receptors. [9] The cells were co-incubated with the conjugates, then after wash-out the cells were irradiated with $635 \mathrm{~nm}$. MTT assay was performed after $72 \mathrm{~h}$.

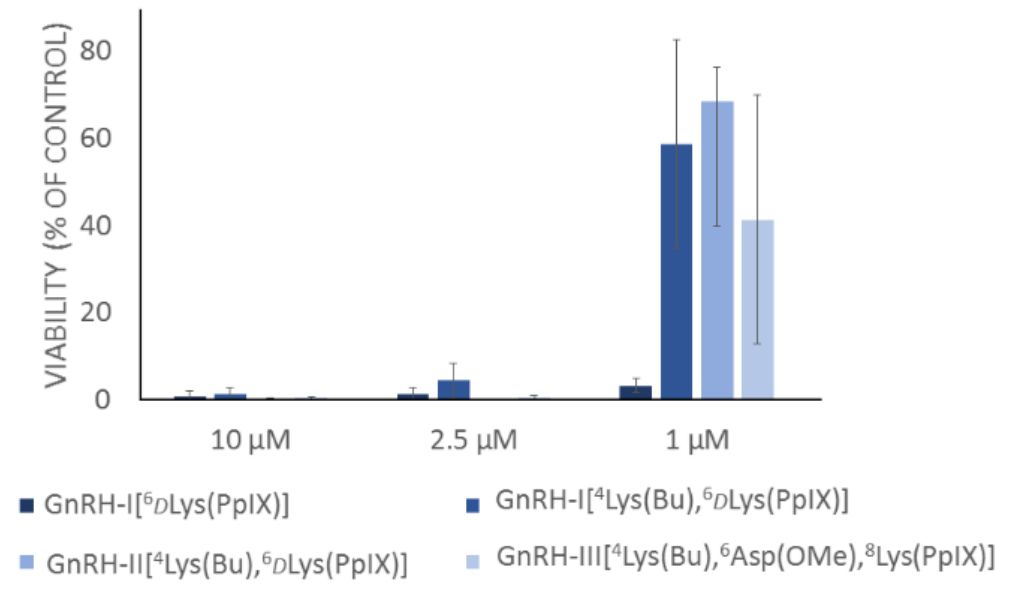

Figure 2: In vitro cytotoxicity of the GnRH-PpIX conjugates

We have investigated the compounds with and without irradiation and we found that the compounds alone, without irradiation, were not toxic in the assays $(>10 \mu \mathrm{M})$. We could also show that a short irradiation time $(10 \mathrm{~min})$ is enough for the tests, since the compounds showed the same in vitro effect with 30 min than with $10 \mathrm{~min}$ irradiation. The shorter time is more beneficial for the further in vivo treatments. Furthermore, we found that all GnRH-Ppconjugates showed excellent anti-tumor effect at low concentrations $(\sim 1 \mu \mathrm{M})$, the best conjugate was GnRH-I[ ${ }^{6} \mathrm{D}$-Lys(PpIX)] (Figure 2).

\section{Acknowledgements}

This project has received funding from the National Research, Development and Innovation Office (NKFIH K119552, NVKP_16-1-2016-0036), Hungary. 


\section{References}

1. N. Malatesti, I. Munitic, I. Jurak, Biophys Rev. 2017 9. 149-168. doi: 10.1007/s12551-017-0257-7

2. A. Nagy, A.V. Schally, Biol. Reprod. 2005 73(5). 851-859. doi: 10.1095/biolreprod.105.043489

3. G. Mezó, M. Manea, I. Szabó, B. Vincze, M. Kovács, Curr. Med. Chem. 2008 15. 2366-2379. doi: $10.2174 / 092986708785909157$

4. R.P. Millar, Anim. Reprod. Sci. 2005 88(1-2). 5-28. doi: 10.1016/j.anireprosci.2005.05.032

5. G. Mezó, M. Manea, Expert Opin. Ther. Patents 2009 19(12). 1771-1785. doi: 10.1517/13543770903410237

6. T. Janáky et al., PNAS 1992 89(3). 972-976. doi: 10.1073/pnas.89.3.972

7. S. Rahimipour, N. Ben-Aroya, K. Ziv, A. Chen, M. Fridkin, Y. Koch, J. Med. Chem. 2003 46(19). 3965-3974. doi: $10.1021 / j m 020535 y$

8. R. Hegedüs et al., Eur. J. Med. Chem. 2012 56. 155-165. doi: 10.1016/j.ejmech.2012.08.014

9. J. Murányi et al., J. Pept. Sci. 2016 22. 552-560. doi: 10.1002/psc.2904 